\title{
Beratung als Sanktion
}

\section{Ein theoretisches Modell und seine Überprüfung in der Praxis}

\author{
Rainer Göckler
}

Prof. Dr. Rainer Göckler ist Studiengangsleiter für Arbeit, Integration und soziale Sicherung an der Fakultät Sozialwesen der Dualen Hochschule Baden-Württemberg in Stuttgart.

E-Mail goeckler@dhbw-stuttgart.de
Die Mitarbeiter und Mitarbeiterinnen (1), die als persönliche Ansprechpartner oder Fallmanagerinnen in den Arbeitsgemeinschaften (ARGEn) und bei zugelassenen kommunalen Trägern seit 2005 ibre Arbeit in der Grundsicherung für Arbeitsuchende verrichten, sind um ihre Aufgabe oftmals nicht $z u$ beneiden.

Sie sind erste Anlaufstelle für viele ungelösten Schwierigkeiten ihrer Klientel, ordnen und strukturieren die Situation nicht nur nach arbeitsmarktlichen Erfordernissen -, entwickeln Rückkehrstrategien in Beschäftigung, entsperren Strom und Wasser, zahlen Mietrückstände, schaffen Zugang zu sozialen Fachdiensten, sichten Papiere, Unterlagen, Rechnungen, Bescheide, klären auf und versuchen berufsbiografische Daten so zu strukturieren, dass Bewerbungsstrategien möglich werden und Annäherung an arbeitsmarktliche Erfordernisse durch Vermittlung in gezielte Maßnahmen erreicht wird.

Dabei sind sie nicht selten überfordert. In fachlicher Hinsicht durch die Fülle unterschiedlicher Anliegen, die die Menschen an sie herantragen, qualifikatorisch durch die mehrheitlich unzureichende Ausbildung und Qualifizierung sowie physisch und psychisch durch überwiegend belastende Betreuungsrelationen und bürokratische Verfahren. Sie werden nicht selten für persönliches Versagen durch ihre Kunden in die Pflicht genommen und sind auch dadurch im Vergleich zu anderen Verwaltungstätigkeiten höheren Risiken ausgesetzt (vgl. Bundesverband der Unfallkassen, 2009). Sie machen Fehler und nicht selten verlagern sie in ihrer Hilflosigkeit institutionelle Logiken auf betroffene Menschen, die ohnehin mit ihrer Situation hadern oder überfordert sind. Nicht zuletzt arbeiten sie in einem gesellschaftlich aufgeheizten Klima mit geringer Wertschätzung ihrer Arbeit, woran die Politik einen erheblichen Anteil trägt. Die Diskussion um Hartz IV ist geprägt von Begriffen wie Kostenexplosion, Missbrauch und mangelnder Transparenz der Organisation und Abläufe (vgl. Eichhorst/Sesselmeier, 2006; Mosley, 2008.). Dies alles hat nicht dazu beigetragen, die »beraterische Grundstimmung « zu verbessern und eine auf Koproduktion ausgerichtete Zusammenarbeit zu verstärken.

\section{Vorgaben}

In dieser Phase ist es naturgemäß besonders heikel, wenn Beraterinnen neben ihrem unterstützenden Auftrag vom Gesetzgeber ganz bewusst kontrollierende und sanktionierende Aufgaben zugewiesen bekommen. Über die Details in der Ausgestaltung des Gesetzes ist mannigfach berichtet worden, sodass auf die Darstellung der formalen Aspekte hier verzichtet wird.

Im Mittelpunkt der nachfolgenden Ausführungen steht die Frage, ob es für Berater möglich ist, ein zugespitztes doppeltes Mandat auch in den Situationen beraterisch durchzustehen, in denen der Kontroll- und Sanktionsauftrag dominiert.

Der Gesetzgeber verquickt den unterstützenden und Hilfe gewährenden Beratungsauftrag in der Gesetzesbegründung nahtlos mit dem Kontroll- und Sanktionsauftrag. Gleichsam wie von Zauberhand soll diese vertrauensvolle $\mathrm{Zu}$ sammenarbeit zwischen den Betroffenen und den Beratern auch in den schweren Zeiten von Leistungskürzung und Leistungsentzug funktionieren, wenn er festlegt, dass "auch in diesem Fall (...) der Zugang des erwerbsfähigen Hilfebedürftigen zu sonstigen Leistungen zur Eingliederung in Arbeit, also auch zu Beratungsund Betreuungsdienstleistungen, erhalten (bleibt) « (Bundestags-Drucksache 15/1516: 61, auch 47 für Jugendliche).

Der Gesetzgeber verwirft mit dieser Festlegung die seit Jahrzehnten in den tra- 
ditionellen Beratungswissenschaften (Pädagogik, Psychologie, Soziale Arbeit) fest verankerte Position, in der zu den grundlegenden konstitutionellen Bedingungen von Beratung die Prinzipien der Freiwilligkeit, der Ergebnisoffenheit und die freie Verfügbarkeit über die Verwertung der Beratungsergebnisse gehören (vgl. exemplarisch Sickendiek, 2007; Mutzeck 2008).

Allerdings bricht in den letzten Jahren die geschlossene Front derer auf, die erfolgreiche Beratung nur unter dem Postulat der Freiwilligkeit betonen. Zu häufig sind insbesondere die Arbeitskontexte von Fachkräften der Sozialen Arbeit in derartige Zwangssituationen eingebunden (vgl. Conen, 2007; Kähler, 2005). Dieses Strukturdilemma, wie man Interessen der Rechtsgemeinschaft und Partikularinteressen der Nutzer in diesen Kontexten professioneller miteinander versöhnt, harrt seit Langem einer professionalisierten Lösung (vgl. Oevermann, 2000: $71 \mathrm{ff}$.$) .$

\section{Modellentwicklung}

Nachfolgende Ausführungen basieren auf einer Studie, die der Autor 2006/2007 bei verschiedenen Grundsicherungsträgern im gesamten Bundesgebiet durch- führte. Sie bestand aus einer standardisierten Befragung betroffener Kunden ( $\mathrm{N}$ = 39) und handelnder persönlicher Ansprechpartner und Fallmanagerinnen (N = 33) von 17 Grundsicherungsträgern (ohne Optionskommunen). In einer sachlogisch-deduktiven Vorgehensweise wurde zunächst ein theoretischer Rahmen entwickelt, der hier nur grob skizziert werden kann (vgl. ausführlicher Göckler, 2009).

Analysiert man die Bedarfssituationen der betroffenen Menschen in den Arbeitsagenturen und bisherigen Sozialämtern sowie die Anforderungen des Gesetzgebers, so zeigt sich, dass die Beratungslandschaft hierfür Beratungsangebote im Feld Bildung und Beruf, Arbeit und Unternehmen, Soziale Arbeit, Verwaltungshandeln/Rechtswissenschaft und Zwangskontexte bereithält. Hinter den jeweiligen Beratungsfeldern lassen sich teilweise spezifische, teilweise unspezifisch übergreifende theoretische Ansätze heranziehen, die auf die Frage zu prüfen waren, welche Angebote (Haltungen, Methoden, Techniken) sie in einer vergleichbaren Situation für ihre Beraterinnen bereitstellen. Insgesamt flossen 17 beratungstheoretische Grundlagen ein, ergänzt um zentrale Positionen »guten und sachgerechten Verwaltungshandelns «.
Daraus ließen sich durch Systematisierung, Bündelung, Hierarchisierung und vereinfachte Codierungsprozesse 20 idealtypische Beratungsinterventionen gewinnen, die den Kern beraterischen Handelns im Sanktionskontext repräsentieren sollen und die insgesamt fünf Managementfunktionen zugeordnet wurden. Diese Größe ermöglichte eine tragfähige Ratinganalyse (2) der aufgezeichneten Gespräche. Dabei werden selbstverständlich nicht immer alle Prüfkriterien in einem Gespräch gleichzeitig relevant. Inhalt, Personen und Dynamik des Gespräches lenken den beraterischen Fokus sehr unterschiedlich.

\section{Beratungsinterventionen}

"Managen « wird hier verstanden als verantwortliche Planung, Gestaltung, Wahrnehmung und Kontrolle eines »Aufgabensets «, das im Rahmen eines Sanktionsgespräches anfällt. Im Gegensatz zur Expansion des Begriffs »Management « und "managen « erscheint er in diesem Zuschnitt angebracht, denn die meisten Funktionen sind durch eigene beraterische Ressourcen gestaltbar. Sieht man von der Bereitstellung der sozial- und arbeitsmarktintegrativen Ressourcen ein-

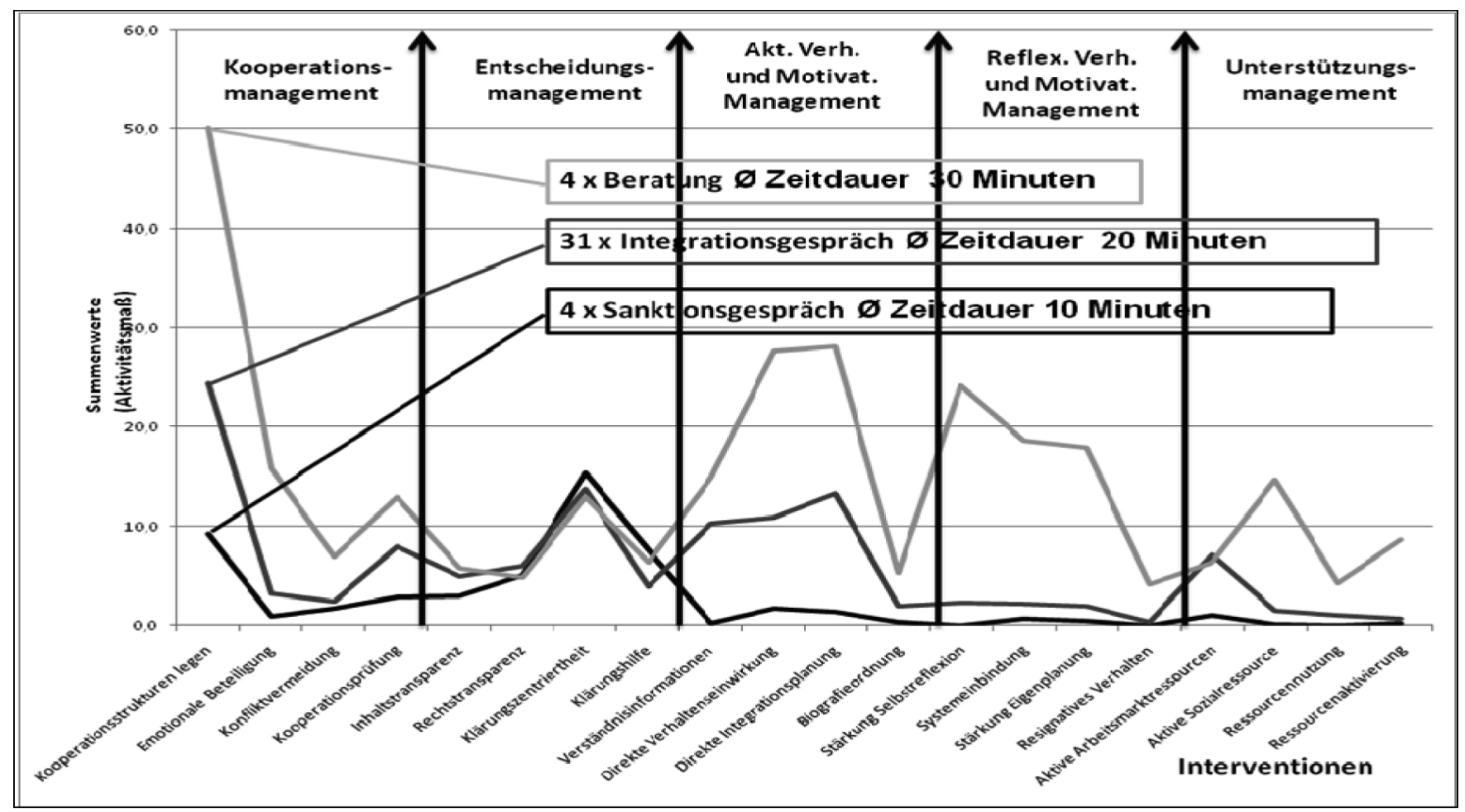

In Gesprächen zwischen persönlichen Ansprechpartnern und Arbeitssuchenden dominieren nach einer faktorenanalytischen Studie drei Gesprächstypen: »Integrationsgespräche«, »Sanktionsgespräche« und »Beratungsgespräche«. 
mal ab, sind alle anderen Funktionen direkter beraterischer Nutzung zugänglich, entsprechendes Bewusstsein und qualifikatorische Güte vorausgesetzt. Zu den einzelnen beraterischen Aufgaben, die sich aus der Modellierung ergaben, nachfolgend ein Überblick:

- Im Aufgabenfeld »Kooperationsmanagement « entwickeln die Beraterinnen angemessene Kooperationsstrukturen zu ihren Kundinnen, greifen auch emotionale Aspekte des Geschehens auf beiden Seiten auf, greifen bei Notwendigkeit auf konfliktvermeidende Gesprächsmethoden zurück und thematisieren verweigerndes Verhalten frühzeitig.

- Im Aufgabenfeld »Entscheidungsmanagement « werden die rechtsstaatlichen Funktionen gebündelt, die zur Entscheidung über eine leistungsrechtliche Sanktion führen. Hierzu gehört die Aufgabe inhaltlich die Zielsetzung der abgesprochenen Angebote zu verdeutlichen, den formalen Notwendigkeiten einer Anhörung Rechnung zu tragen (Rechtstransparenz), eine gezielte und nachhaltige Aufklärung der Umstände herbeizuführen sowie angemessene Unterstützung bei entlastenden Formulierungen zu geben.

- Im Aufgabenfeld des »aktiven Verhaltens- und Motivationsmanagements " geben Beraterinnen aktive Informationen zu übergreifenden Aspekten des Sanktionskontextes. Sie nutzen zudem die Thematik zur gezielten Verhaltensbeeinflussung, bearbeiten irrationale Überzeugungen und stärken prosoziales Verhalten. Sie konfrontieren Kunden mit den Folgen ihres Verhaltens und erneuern die aktuelle Integrationsstrategie. Dabei nutzen Sie die Chance, das bisherige Bildungs- und Berufsgeschehen zu prüfen.

- Im Aufgabenfeld des »reflexiven Verhaltens- und Motivationsmanagements « gelingt es dem Berater, Elemente einer adäquaten Selbsterfahrung und Selbstreflexion zu stärken. Er fördert systematisch rückbezügliche Fragen zum gezeigten Verhalten und stärkt prosoziale und sanktionsvermeidende Überlegungen. Personen und Institutionen, die im Kontext des Sanktionsgeschehens Bedeutung haben oder für die weiteren Integrationsplanungen von Bedeutung sind, werden vom Berater systematisch einbezogen. Er fördert Eigenplanung und eigene Realisierungs- überlegungen. Erscheinungsformen resignativen Verhaltens greift er auf und reflektiert diese mit dem Kunden.

- Das Aufgabenfeld »Unterstützungsmanagement « ist Kennzeichen der sozialpolitischen Einbettung der Aufgabe. Die Beraterin bietet konkrete arbeitsmarktbezogene Ressourcen an, die dazu beitragen können, die Folgen einer Sanktion zu minimieren und den Fall weiter zu entwickeln. Dies gilt auch für entsprechende sozial-integrative Angebote. Der Berater prüft mit dem Kunden zur Verfügung stehende Ressourcen und Netzwerke, regt zu ihrer Nutzung an bzw. entwickelt entsprechende Strategien mit der Kundin.

\section{Anwendung}

Insgesamt darf man erwarten, dass diese Managementfunktionen mit insgesamt 20 Prüfitems die notwendigen Aktivitäten abbilden, die im Kontext des Kontrollund Sanktionsauftrages eine beraterische Anschlussfähigkeit sichern können, ohne dass diese alle im Einzelfall immer relevant werden. Auch wenn ein Sanktionskontext kaum vertrauensförderliche Bedingungen erhält, ist die weitere und außerhalb des rein rechtlichen Bezugsrahmens stattfindende Aufarbeitung der Sanktionsumstände und Sanktionsfolgen die einzige Möglichkeit, eine beratungsbezogene Anschlusskooperation zu sichern.

Die Analyse der Gespräche erfolgte nach einem vereinfachten Modell in Anlehnung an das » Ratinginventar Lösungsorientierter Interventionen (RLI)« (vgl. Honermann et.al. 1999). Im vorliegenden Fall wurden alle Gespräche in Minuteneinheiten transkribiert. Für jede Minute waren danach alle Items auf einer Skala von 0 (es liegen keine Aktivitäten vor) bis 3 (Aktivität sehr stark/intensiv) einzuschätzen.

\section{Ergebnisse}

In insgesamt 39 sogenannten Sanktionsgesprächen wurde das Modell zur Erfassung der einzelnen Items eingesetzt. Ein faktorenanalytisches Verfahren ermöglichte eine relativ eindeutige Exploration von drei Gesprächstypen (vgl. Abb. Seite 97). Im Ergebnis zeigt sich eine
Häufung bei der Gesprächsform »Integrationsgespräch «, der rund achtzig Prozent $(\mathrm{N}=31)$ der bewerteten Gespräche zuzurechnen sind. Die beiden anderen Gesprächstypen (»Sanktionsgespräche« und »Beratung «) waren mit jeweils vier Zuordnungen deutlich unterrepräsentiert. Die wesentlichen Kennzeichen der Gesprächstypen lassen sich folgendermaßen interpretieren:

- Sanktionsgespräche sind dadurch gekennzeichnet, dass sie ihren zentralen Fokus auf die administrative Abarbeitung des Sanktionskontextes legen. Sie spiegeln ein rein hoheitliches Verständnis der Grundsicherungsaufgabe wider, das sicherlich für eine moderne Dienstleistungsbehörde als nicht mehr zeitgemäß zu betrachten ist. Beraterische Aspekte, die über den Sanktionskontext hinausreichen, werden in ihnen weitgehend ausgeblendet. Sanktionsgespräche können im Regelfall nicht als beraterische Dienstleistung identifiziert werden.

- Integrationsgespräche fokussieren in ihrem Arbeitsverständnis auf eine rasche und überwiegend eher direktive Erledigung der anfallenden Aufgabe. Auch wenn der Kooperationsbeziehung zum Kunden große Bedeutung beigemessen wird, betrifft diese doch eher die Absicherung der eigenen Vorgehensweise. Der Fokus liegt weniger auf einer Stärkung eigener Autonomiepotenziale, sondern auf vermittlungsorientierten Vorgehensweisen und einer mehr oder weniger direktiven Form der Verhaltensvorgaben, flankiert vom Angebot standardisierter Unterstützungsmaßnahmen. Es liegt nahe, diese Befunde dahingehend zu interpretieren, dass sie die mehrheitlich pragmatische Antwort der Mitarbeiter auf zu hohe Kundenbetreuungszahlen und eine einseitig verkürzte geschäftspolitische Ausrichtung auf reine Vermittlungserfolge darstellen. Auch hier sind Konstellationen denkbar, in denen ein derartiger Gesprächstyp sinnvoll sein kann. Dabei ist grundsätzlich zu bemängeln, dass über das Verfahren und die beraterischen Vorgehensweisen im Vorfeld keine Verständigung mit dem Kunden gesucht wurde. Ziele der Kunden werden innerhalb des Gespräches eher zufällig abgerufen und eine Vereinbarung über die im Gespräch zu erarbeitenden Ziele unterbleibt weitgehend. Insofern sind 
wesentliche Indikatoren, die einen Beratungsprozess konstituieren, bei Integrationsgesprächen nicht gegeben. Auch wenn der Anlass gesetzgeberisch vorgezeichnet wird, von daher immer integraler Bestandteil der Beratungsinhalte sein muss, reicht der gesetzgeberische Auftrag der Unterstützung und Beratung über den Sanktionskontext hinaus.

- Der Gesprächstyp Beratung in dieser Untersuchung zeichnet sich durch eine gleichmäßigere Wahrnehmung aller Managementfunktionen aus. Der Sanktionsauftrag wird nicht negiert, jedoch eingebettet in weitere Überlegungen zur Situation des Betroffenen und den Möglichkeiten, hieraus zukunftsfähige Perspektiven zu gewinnen. Das mag nicht immer gelingen, dennoch ist auffällig, dass alle als »Beratung « typisierten Formen eine zielgerichtete Weiterentwicklung des Falles anstreben. Beratung in diesem Sinne ist demnach alles andere als konturlos und sich vor konkreter Ergebnisverantwortung drückend. In kaum einem anderen der aufgezeichneten Sachverhalte ist die Weiterentwicklung des Falles so greifbar, wie in diesen Beratungsfor- men. Es fällt eben leichter zu konkreten Vereinbarungen zu kommen, wenn man gemeinsam an einem Ziel gearbeitet hat.

\section{Wirkungen}

Eine weitere wissenschaftliche Fundierung dieser explorativen Studie müsste mit unabhängigen Vergleichsgruppen arbeiten, die Konstruktvalidität stärker absichern, Kriterien erarbeiten, was als »Erfolg « gemessen werden sollte und dies in einer mehrstufigen (6 Monate, 12 Monate) Nachbefragung absichern.

Dennoch ergab die nachgehende Befragung der betroffenen Kunden mehrere klare Hinweise auf die Verständlichkeit, Akzeptanz und Nachhaltigkeit einer guten Beratung gegenüber den anderen Gesprächsformen. Dies betraf vor allem das Verständnis des Sanktionsgeschehens, die Einbeziehung der Kundenäußerungen und ihrer Sichtweisen sowie die Akzeptanz des Gesprächspartners.

Das Modell könnte als Rahmen fungieren, mit der man öffentliche Beratung in Zwangskontexten - und vielleicht nicht nur dort - für grundlegende Beratungs-

\section{Literatur}

Bundesverband der Unfallkassen (2009): Arbeitsbelastungen und -bedrohungen in Arbeitsgemeinschaften nach Hartz IV (ABBA). Zwischenbericht. Unter: (Zugriff 12/2009) http://www.dguv.de/inhalt/praevention/aktionen/abba_projekt/index.jsp.

Conen, Marie-Lusie, Cechin, Gianfranco (2007): Wie kann ich Ihnen helfen, mich wieder loszuwerden? Therapie und Beratung in Zwangskontexten. Carl-Auer Verlag, Heidelberg.

Göckler, Rainer (2009): Beratung im Sanktionskontext. Sanktionsgespräche in der Grundsicherung für Arbeitsuchende. Theorie und Praxis der Umsetzung. dgvt-Verlag, Tübingen.

Hausinger, Brigitte (2009): Umrisse einer Beratungswissenschaft. In: Möller, H., Hausinger, B. (Hg.); Quo vadis Beratungswissenschaft? VS Verlag; Wiesbaden, S. $177-184$.

Honermann, Hermann, Müssen, Peter, Brinkmann, Andrea, Schiepek, Günter (1999): Ratinginventar Lösungsorientierter Interventionen (RLI). Ein bildgebendes Verfahren zur Darstellung ressourcen- und lösungsorientierten Therapeutenverhaltens. Verlag Vandenhoeck\&Ruprecht, Göttingen.

Mosley, Hugh (2008): Das Hartz-IV-Experiment. In: Wirtschaftsdienst, 2, S. 90-95.

Mutzeck, Wolfgang (2008): Kooperative Beratung. Grundlagen, Methoden, Training, Effektivität. Beltz Verlag (6. Aufl.), Weinheim, Basel.

Oevermann, Ulrich (2000): Dienstleistung der Sozialbürokratie aus professionalisierungstheoretischer Sicht. In: Harrach, E.-M., Loer, T., Schmidtke, O.: Verwaltung des Sozialen. Formen subjektiver Bewältigung eines Strukturkonflikts. UVK, Konstanz, S. 57-78. aufgaben anschlussfähig gestalten kann. Die Vielfalt der Anliegen benötigt ein eklektizistisches Beratungsverständnis, welches die Mitarbeiter in die Lage versetzt, die herangetragenen Bedarfslagen aufzunehmen, zu erkennen und zu strukturieren. Nur in den Fällen unmittelbarer arbeitsmarktintegrativer und leistungssichernder Aufgaben ist es ihnen möglich, auf die Bedarfslagen mit eigenen materiellen und Beratungsressourcen zu reagieren. In den meisten anderen Fällen benötigen sie die Unterstützung von Fachkräften, die gezielter auf Bedarfslagen der Menschen eingehen können.

Genau hier verläuft die Schnittstelle zwischen guter öffentlicher Beratungsarbeit in Behörden und der Beratungsarbeit von professionellen Experten aus Sozialer Arbeit, Medizin oder Therapie. Dies wäre Aufgabe der öffentlichen Träger und ihrer Ausbildungseinrichtungen, ein Beratungskonzept vorzulegen, das eine eigenständige Professionalisierung der öffentlichen Beratung ermöglicht und gleichzeitig die Kompetenzen der externen Profis systematisch mit reflektiert.

So wäre allen geholfen: den Fallmanagern und persönlichen Ansprechpartnern in der Grundsicherung (auch in anderen behördlichen Zwangskontexten), die endlich über ein eigenes professionalisiertes Beratungsverständnis verfügten und in den » Beratungswissenschaften « (Hausinger, 2009) damit wahrnehmbar wären, den vielen freien und karitativen Beraterinnen, die an dieser Nahtstelle ihren spezialisierten Beratungsauftrag wahrnehmen könnten, und den betroffenen Menschen, die in einem geklärten Prozess die Hilfen bekommen, die sie tatsächlich benötigen.

\section{Anmerkungen}

(1) Nachfolgend verwende ich abwechselnd mal männliche, mal weibliche Bezeichnungen, um den Genderaspekten Rechnung zu tragen.

(2) Das von Honermann et al. (1999) vorgelegte Ratinginventar, das für die Untersuchung Pate stand, umfasst einen Kriterienkatalog von 23 Items. 